\title{
Live Video Segmentation in Dynamic Backgrounds Using Thermal Vision
}

\author{
Viet-Quoc Pham ${ }^{1}$, Keita Takahashi ${ }^{2}$, and Takeshi Naemura ${ }^{1}$ \\ ${ }^{1}$ Graduate School of Information Science and Technology, The University of Tokyo \\ ${ }^{2}$ IRT Research Initiative, The University of Tokyo \\ Hongo 7-3-1, Bunkyo-ku, Tokyo, 113-8656 Japan \\ \{viet, keita, naemura\} @hc.ic.i.u-tokyo.ac.jp \\ http://hc.ic.i.u-tokyo.ac.jp
}

\begin{abstract}
In this paper we describe a new technique for live video segmentation of human regions from dynamic backgrounds. Correct segmentations are produced in real-time even in severe background changes caused by camera movement and illumination changes. There are three key contributions. The first contribution is the employing of the thermal cue which proves to be very effective when fused with color. Second, we propose a new speed-up GraphCut algorithm by combining with the Bayesian estimation. The third contribution is a novel online learning method using accumulative histograms. The segmentation accuracy and speed are quite capable of the live video segmentation purpose.
\end{abstract}

Keywords: Live video segmentation, infrared image sensors, GraphCut.

\section{Introduction}

This paper addresses the problem of live video segmentation of human regions from dynamic backgrounds. Different from offline video segmentation that can use the whole video as the reference for the segmentation, live video segmentation does not employ future information, so that the estimation should be based only on the past frames. A prime application is live background substitution in broadcasting and teleconferencing. This demands layer separation with high quality and computational efficiency sufficient to attain live streaming speed. Besides, it must deal with dynamic background changes caused by several factors such as camera movement, illumination changes, etc. However, by using only monocular video, even most effective recent researches [1,2 can at most deal with small camera shakes. Therefore, segmentation with more severe background changes like camera movement requires more effective cues besides color and motion.

An impressive decrease in both size and price of thermal vision cameras promises their wide applications in both researches and daily life. Aiming to achieve live video segmentation with higher accuracy, our research group has been developing a unique system named "thermo-key" [3] which combines a color camera and a thermal vision camera. Both cameras share the same optical axis using an IR mirror as shown in Fig. 1, so that each pixel on the combined image

T. Wada, F. Huang, and S. Lin (Eds.): PSIVT 2009, LNCS 5414, pp. 143-154, 2009.

(C) Springer-Verlag Berlin Heidelberg 2009 


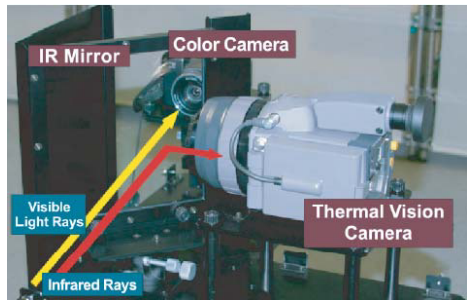

Fig. 1. Camera system for thermo-key

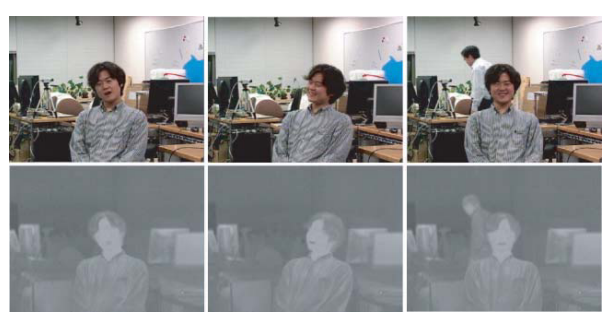

Fig. 2. Captured sequences from the thermo-key system: first row- color sequence, second row- thermo sequence

has a thermal value as well as a color value. Sample sequences captured from this system are shown in Fig. 2. In this paper, we will prove that by using this thermal cue, we can attain more accurate segmentation results than [1] even when using a simpler probabilistic segmentation model.

We propose three main contributions in this paper. The first one is the employing of the thermal cue which proves to be very effective when fused with color. Second, we propose a new speed-up GraphCut algorithm by combining with the Bayesian estimation. The advantage of GraphCut is that it can simultaneously consider texture (color+thermo) information and edge (contrast) information in its optimization scheme. It has been adopted for interactive image segmentation [415] and automatic video segmentation [12]. Our method aims to realize accurate human-region segmentation with lower complexity than [1 by combining the Bayesian estimation approach with a GraphCut algorithm. The third contribution is a novel online learning method using accumulative histograms which can be deal with background changes.

In this paper, previous works in video segmentation are described in Section 2 Section 3 introduces our proposed algorithm. Experiments and conclusions are presented in Section 4 and 5 .

\section{Related Works}

Classical image segmentation tools use either texture (colour) information, e.g. Magic Wand [6], or edge (contrast) information, e.g. Intelligent Scissors [7]. Recently, an approach based on optimisation by GraphCut has been developed which successfully combines both types of information. Boykov et al. 4 and Rother et al. [5] introduced powerful optimisation techniques using GraphCut for interactive segmentation. Although color/contrast cues have been demonstrated to be very effective for interactive segmentation, they alone are still insufficient for fully automatic segmentation. This suggests a need for a robust approach that fuses a variety of cues, for example depth, motion, and so on.

In [2, segmentation for static background was performed by combining background subtraction with the GraphCut optimization. Criminisi et al. 1] and Kolmogorov et al. [8] described algorithms capable of real-time segmentation of 


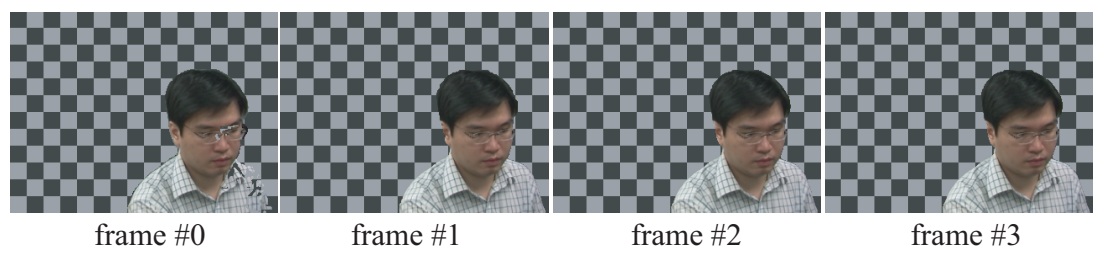

Fig. 3. In the segmentation result of the first frame, some errors appear because some parts of the object person have temperatures closed to the background temperature. However, these errors are disappeared in the next frames thanks to our robust segmentation method.

foreground from background layers in video sequences, in which colour, contrast and another key feature were fused to infer layers. The key feature used in [1] was motion, so that their algorithm could be applied to monocular videos, while Kolmogorov et al. 8] employed the depth feature obtained from stereo videos.

As stated before, the above researches can at most deal with small camera shakes. Segmentation with more severe background changes caused by camera movement requires more effective cues besides color and motion. The purpose of this research is to fuse color, contrast with temperature to realize the real-time segmentation. Our work is an extension as well as an improvement of the previous work [3] which was based on a simple threshold processing to the thermal images (see Fig. 8).

\section{Segmentation Algorithm}

\subsection{Assumption}

The only constraint for our live video segmentation system is the requiring of the background of the first frame. This simple assumption is reasonable because in most filming works, the background is kept fixed before shooting. This constraint helps us to quickly detect the foreground region from the background for the first frame by a simple background subtraction method. We must notice that the background subtraction is applied for only the first frame. After starting capturing, the camera can move freely, making the background changes, and therefore, the background subtraction will be no more in use.

The background subtraction can be performed on both color and thermal channels, however, only thermal channel is enough to produce rather good results. Although it sometimes does not work well due to the closed temperatures in some places between the foreground and the background, but by applying our robust segmentation algorithm, the segmentation errors appeared in the first frame will be removed in the next frames (see Fig. 3).

Some researches like [1] did not require background for the first frame, but they used the motion properties instead. When the object moves while the background is kept static, the moving parts are detected and segmented. Much time was needed until the whole object is segmented (your hand will not be segmented 


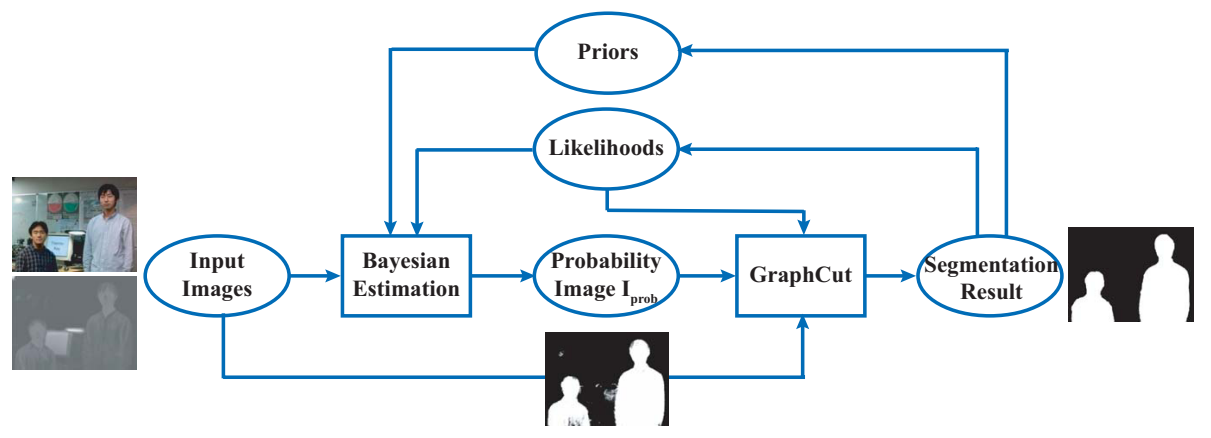

Fig. 4. Demonstration for the two-step segmentation algorithm

until you move it). Furthermore, offline learning of motion properties was needed in some cases. For the live video segmentation purpose, it is clearly less practical than our assumption.

The segmentation algorithm for the next frames is stated in the successive sections.

\subsection{Algorithm Overview}

Figure 4 illustrates the entire algorithm. Our algorithm takes the color and thermal images captured by our thermo-key system as the inputs, and outputs the segmentation result (a binary mask) of the foreground region.

The first originality of our algorithm is the employing of the thermal feature combining with the color feature. Second, we propose a probabilistic model combining the Bayesian estimation approach with a GraphCut algorithm. Most GraphCut based segmentation methods including [1] created the graph model for all image pixels. Such methods cost time because the calculation cost of the GraphCut algorithm relies mostly on the number of the graph nodes. Our contribution is that before applying GraphCut, we perform a pre-GraphCut step, or a trimap making step to reduce the unknown region (i.e. the region around the object boundary that is still unknown to be of the background or the foreground class). As a result, we can speed up the GraphCut process. The third originality is a novel online learning method using accumulative histograms which can be deal with background changes. These two steps of segmentation and online learning method are stated in the next sections.

\subsection{Step 1- Pre-GraphCut Step}

The first step calculates the probability of each pixel to be in the foreground based on the Bayesian estimation. This calculation can be performed in the linear time. After that, the results are used to make the trimap.

For details, let $C_{i}^{t}$ and $T_{i}^{t}$ be the color and thermal values of the $i^{t h}$ pixel on the combined image at period $t$ captured by the thermo-key system. Based on the Bayes' formula, the probability of this pixel to be in the foreground is given as 


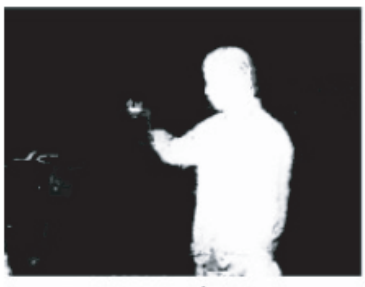

$\boldsymbol{\alpha}^{t-1}$

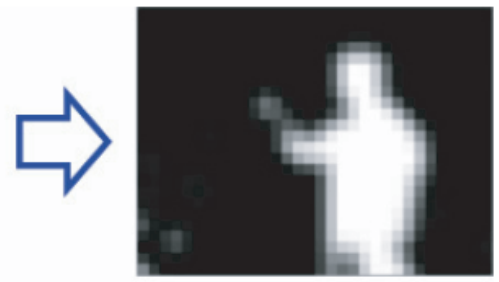

$\mathbf{M}^{t}$

Fig. 5. The smoothed map is created from the segmentation result of the previous frame

$$
P^{t}\left(F \mid C_{i}, T_{i}\right)=\frac{P^{t}\left(C_{i}, T_{i} \mid F\right) P_{i}^{t}(F)}{P^{t}\left(C_{i}, T_{i} \mid F\right) P_{i}^{t}(F)+P^{t}\left(C_{i}, T_{i} \mid B\right) P_{i}^{t}(B)}
$$

where $P(*)$ represents the probability of "*". $F$ and $B$ are the foreground and background respectively, into which each pixel should be classified. The posterior probability, $P^{t}\left(F \mid C_{i}, T_{i}\right)$, is represented as the product of the likelihood $P^{t}\left(C_{i}, T_{i} \mid F\right)$, and the prior probability, $P_{i}^{t}(F)$. The likelihood is calculated from the histogram which was constructed from previous segmentation results. Since there are some correlations between color and thermal information, we should use a 4-D accumulative histogram, where 3 dimensions are assigned for YUV color channels, and another dimension is for the thermal channel. The meaning of accumulative histogram is explained in section 3.5 .

The prior probability is calculated from the previous image frame since successive frames in the temporal domain would have strong correlations. To be more precise, let $\alpha^{t-1}$ be the 2-D mask of the segmentation result (taking 255 for $F$ and 0 for $B$ ) at period $t-1$, and $M^{t}$ be the smoothed map of $\alpha^{t-1}$ which is defined as follows

$$
M^{t}=G_{7 \times 7}\left(\operatorname{Resize}_{\times 10}\left(G_{3 \times 3}\left(\operatorname{Resize}_{\times 1 / 10}\left(\alpha^{t-1}\right)\right)\right)\right) / 255
$$

where $G$ is the Gaussian filter and Resize is the scaling transformation. Then the prior probability $P_{i}^{t}(x)$ can be inferred from $M^{t}$

$$
P_{i}^{t}(x)= \begin{cases}M_{i}^{t} & (x=F) \\ 1-M_{i}^{t} & (x=B)\end{cases}
$$

An image of a smoothed map is shown in Fig. 5.

\subsection{Step 2- GraphCut Optimization}

The set of posterior probabilities $P^{t}\left(F \mid C_{i}, T_{i}\right)$ over all image pixels $i$ forms the probability map $I_{\text {prob }}$. We create the trimap, $\operatorname{Tr}(i)$, which takes one of the three values $\{\mathrm{F}$ (foreground), B (background), and $\mathrm{U}$ (unknown) $\}$, based on the value of $I_{\text {prob }}(i)$

$$
\begin{cases}\text { if }\left(I_{\text {prob }}(i)<\epsilon\right): & \operatorname{Tr}(i)=B \\ \text { if }\left(I_{\text {prob }}(i)>1-\epsilon\right): & \operatorname{Tr}(i)=F \\ \text { otherwise : } & \operatorname{Tr}(i)=U\end{cases}
$$




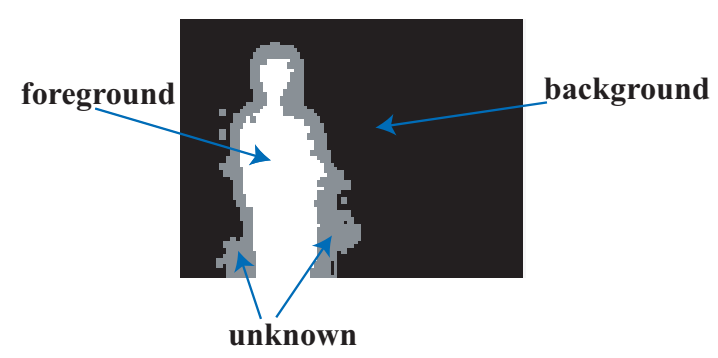

Fig. 6. A trimap is a classification of an image into three regions: foreground (white), background (black) and unknown (gray)

where $\epsilon$ is a small real value. Then, a filter is applied to the trimap to remove noises, since clusters of noise pixels seem to be relatively small in size. And this filter operation can be achieved in linear time. An image of a trimap is shown in Fig. 6.

Before getting further into our proposed method, it is better to explain briefly about the GraphCut optimization algorithm [4]. Let $S e g$ be a segmentation of the image where $\operatorname{Seg}(\mathrm{i})$ takes $F$ (Foreground) or $B$ (Background) for the $i^{\text {th }}$ pixel. We define the energy function by

$$
\mathrm{E}(S e g)=\operatorname{Data}(S e g)+\lambda \operatorname{Sth}(S e g)
$$

where the data term $\operatorname{Data}(\mathrm{Seg})$ evaluates the pixel-wise costs, and the smoothness term $\operatorname{Sth}(S e g)$ evaluates the inter-pixel costs. $\lambda$ is a weighting coefficient. This function can be minimized by the GraphCut algorithm, and the optimization solution should produce a good segmentation because it keeps the balance between the region property and the boundary property of the segments.

In our method, the above filtered trimap is set as the initial values for the GraphCut optimization. As we can see from Fig. 6, the unknown region is much smaller than the whole image. Because in our proposed algorithm, the GraphCut process is applied only on the unknown region, its calculation speed can increase considerably.

We define the data term as the weighted sum of the likelihood of the color and thermal values $L\left(C_{i}, T_{i} \mid \operatorname{Seg}(i)\right)$, and the probability map given by the first step $I_{\text {prob }}(i)$

$$
\begin{aligned}
\operatorname{Data}(\operatorname{Seg}) & =(1-\mu) \sum_{i}-\log \left(L\left(C_{i}, T_{i} \mid \operatorname{Seg}(i)\right)\right) \\
& +\mu \sum_{i}-\log \left(I_{\text {prob }}(i)\right)
\end{aligned}
$$

where the likelihood can be obtained from the accumulative histograms (section 3.5). We employ the probability map $I_{\text {prob }}$ here because it is a good estimation which combines both the temporal priors and the likelihoods. This is another different point of our proposed method from the others. 


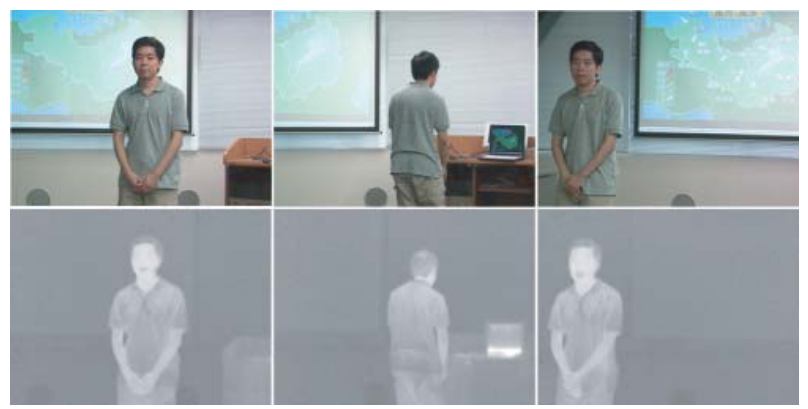

Fig. 7. When the camera moves, the textures of the background change noticeably. A new object (a laptop PC) appears in the second image, and the illuminations in the first and third images are clearly different.

To set the smoothness term, we use an ad-hoc function which was used by most other methods

$$
\operatorname{Sth}(\operatorname{Seg})=\sum_{(i, j) \in N}[\operatorname{Seg}(i) \neq \operatorname{Seg}(j)] \frac{e^{-\left\|C_{i}-C_{j}\right\|^{2} /\left(2 \sigma^{2}\right)}}{\|\operatorname{dist}(i, j)\|}
$$

where $N$ represents all neighborhood pixel pairs, and dist $(i, j)$ denotes Euclidean distance between $i^{\text {th }}$ and $j^{\text {th }}$ pixels. $\sigma$ can be estimated as "camera noise". This function imposes larger cost to the discontinuous regions with little color variations. Our implementation of the GraphCut algorithm uses the minimumcut algorithm from [9].

\subsection{Accumulative Histogram}

The other contribution of our proposed method is the new idea of the accumulative histogram. The main problem of segmentation from dynamic backgrounds is how to deal with the circumstance where the textures of the foreground and background are changing along the time (see Fig. (7). One of the solutions is to update the likelihood, or the 4D histograms of the color and thermal values. Here we creates two accumulative histograms $\mathrm{Hist}_{F}$ and $\mathrm{Hist}_{B}$ for the foreground and background likelihood models, respectively. We update them adaptively over successive frames, based on data from the segmented foreground and background in the previous frame. The new idea of our method is that we do not update all bins of the histograms, but only the new bins (a bin is a certain range of $4 \mathrm{D}$ vectors expressing the color and thermal values). To be more precise, only bins which have the current values of zeros in the both histograms were updated with the new values learned from the previous segmented image. The reason is that segmentation results always include errors, and if we update all bins, these errors will propagate, and as the sequence, both histograms will converge to each others, making the discrimination between the foreground and background becomes impossible. 


\section{Experimental Results}

In our experiment, we captured two video sequences named "Video Chatting" and "Weather Forecast". Each frame in each sequence is a pair of a 24-bit color image and a 8-bit thermal image (both are $320 \times 240$ pixels). In "Video Chatting", a person who is the segmentation object was talking in front of the camera while the background was changing following the movement of the cameras. In "Weather Forecast", the person was moving backward and forward while making some hand gestures during his presentation. The capturing system was slowly panned during the sequence to track the person's position. Besides, some objects like monitors whose temperatures closed to the body temperature were located in the backgrounds, making the backgrounds more dynamic in both color and thermal channels. Therefore, the segmentation task became so difficult that a simple thresholding method based on the thermal information, which was employed in the earlier implementation of the thermo-key system [3], became inapplicable in such circumstances (see Fig. 8).
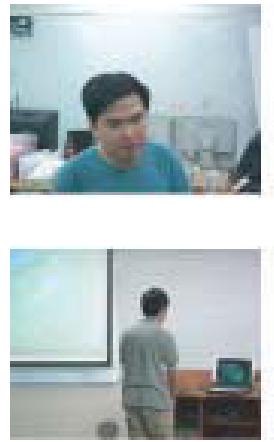

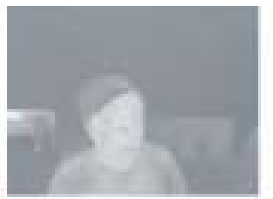

(a)

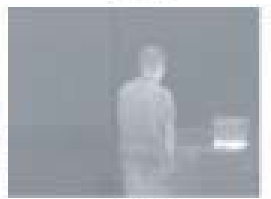

(b)
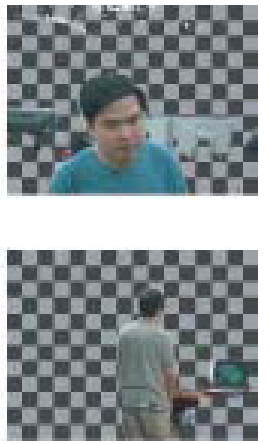

Fig. 8. Results from the thresholding method applied to the 100th frame from sequences (a)"Video Chatting" and (b)"Weather Forecast". From left to right: input color images, input thermal images, thresholding results. Some places in the background whose temperatures are closed to the body temperature were misclassified.

Four coefficients $(\epsilon, \lambda, \mu, \sigma)$ in Section 3.4 were set as $(\epsilon=1 / 32, \lambda=20, \mu=$ $0.75, \sigma=27)$.

\subsection{Segmentation Quality}

Figure 9 shows the segmentation results for some frames from the two sequences. The first and second rows show the input color and thermal sequences captured from the thermo-key system. The third row shows the segmentation results. On the last row, the segmentation results are superimposed on the corresponding color images. The entire results are available from our web site1.

\footnotetext{
1 http://www.hc.ic.i.u-tokyo.ac.jp/ viet/liveseg
} 

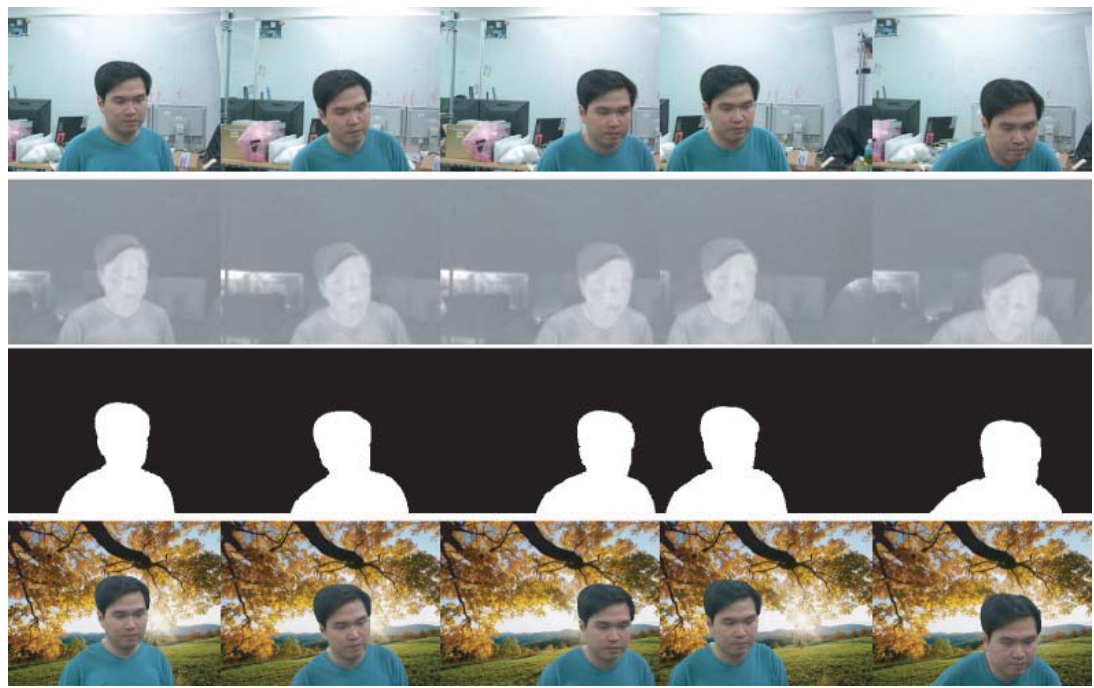

(a) Video Chatting
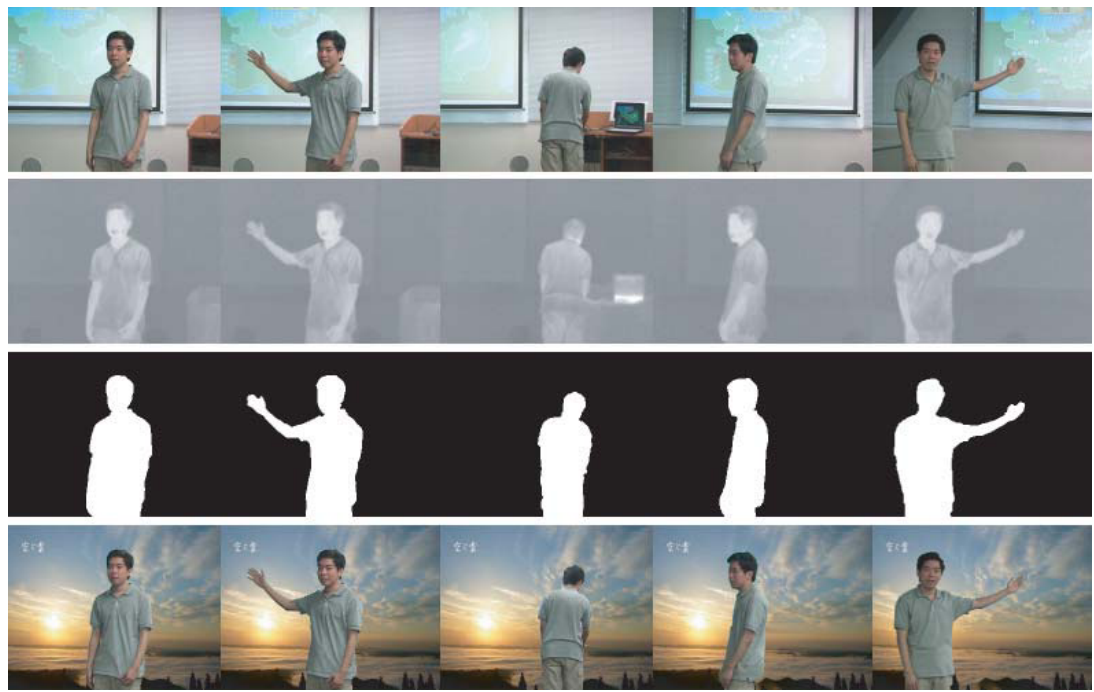

(b) Weather Forecast

Fig. 9. Segmentation results from two sequences (a) "Video Chatting" and (b) "Weather Forecast". First row: input color sequence, second row: input thermal sequence, third row: output segmentation results, last row: background substitution results. Notice that the cameras were panned in both sequences.

To evaluate the results, we first made a comparison between two cases, in which our proposed method was performed with and without using the thermal feature, in order to show the importance of this feature. Second, to prove the effectiveness of our method, we made another comparison between our 


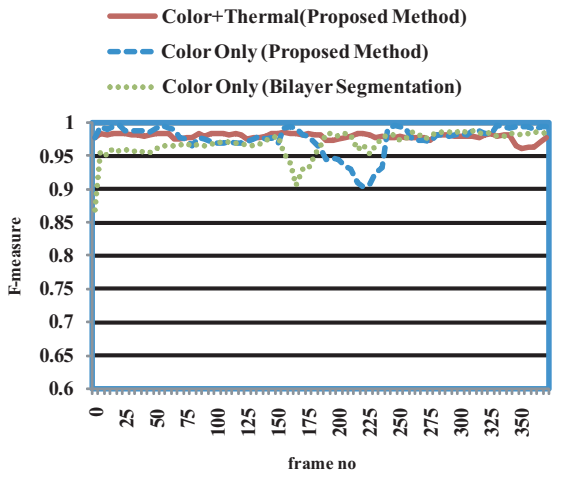

(a)

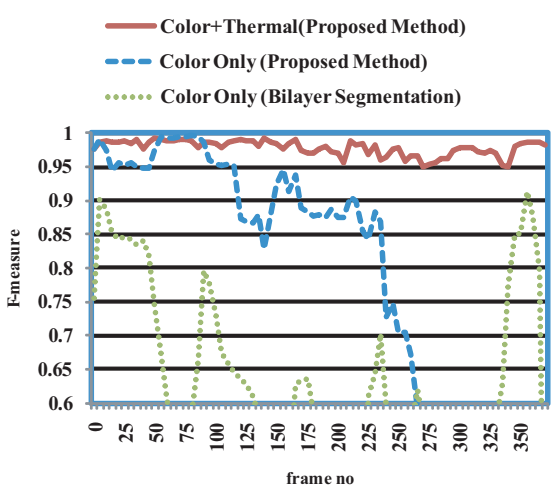

(b)

Fig. 10. F-measures calculated over 370 frames from two sequences (a) "Video Chatting" and (b) "Weather Forecast"

proposed method with one of the most effective conventional methods- Bilayer Segmentation[1]. This method was implemented from the source code provided in 10. The temporal prior and the likelihood for motion in this method were learned from the ground truths of the first 100 frames. To evaluate the quality of the segmentation results, we used F-measure which is the harmonic mean of precision and recall

$$
F=2 \cdot(\text { precision } \cdot \text { recall }) /(\text { precision }+ \text { recall })
$$

The hand-labeled ground truths were used to calculate the precision and recall. We must notice that these ground truths were only used for the evaluation and not employed in our algorithm at all. The graphs of F-measures calculated over 370 frames from the two sequences showed the comparison results (see Fig. 10). In "Video Chatting", where the background textures did not change so much, the differences between the three methods were not so large. However, on an average, our proposed method with using thermal information outperformed the others (its F-measures were always larger than 95\%). In "Weather Forecast", where the background changed noticeablely in both its texture and illumination, the differrences between the three methods became more clear. Bilayer Segmentation did not work in this case because its motion model did not adapt to severe changing backgrounds. Besides, because its algorithm needed to be trained by lots of hand-labeled segmentation data to build the temporal prior, color, and motion models, this method was not capable of the live video segmentation purpose. Another thing which can be learned from the graph result is the effectiveness of using the thermal feature. In such case when the color feature are not reliable due to the camera motion and illumination changes, using the thermal feature is a good solution. The F-measures from our proposed method were always over $95 \%$, a value that is quite capable of the live video segmentation purpose. 

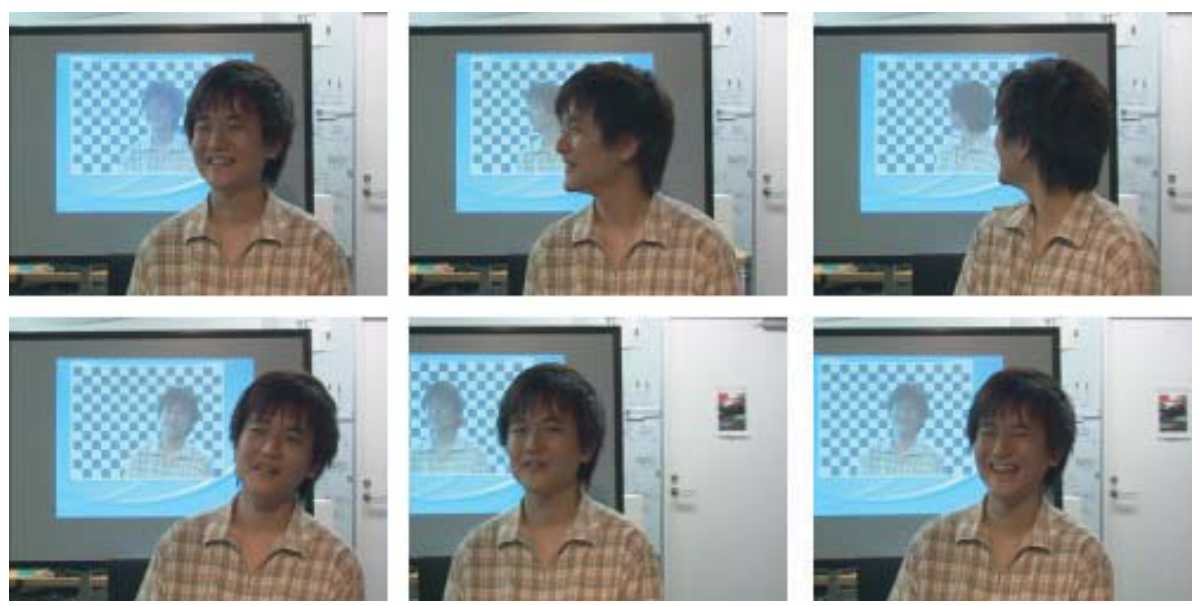

Fig. 11. Live video segmentation demonstration. The segmentation results are displayed lively in the behind projector screen.

\subsection{Calculation Cost}

Our experiment was performed on the computer with Intel Core2 Quad CPU, $2.40 \mathrm{GHz}$ and $4 \mathrm{~GB}$ memory. The calculation time was about 90 milliseconds for each frame. To prove the fact that initializing the human and background regions could speed up the GraphCut process, we also measured the calculation time for the case in which the human and background regions were not initialized, i.e. the GraphCut algorithm was applied over the entire image region. This calculation time was about 200-300 milliseconds for each frame which was much slower than the proposed method.

\subsection{Live Video Segmentation}

In the live video segmentation system, the delay times (the waiting time until the result is outputed) must be kept as short as possible. In our implementation, besides employing the speed-up GraphCut algorithm, we also applied parallel processing for the capturing phase and the segmentation processing phase. As a result, the delay time was decreased to less than 200 milliseconds (about two frames' processing time). Fig. 11 shows our live video segmentation demonstration in which the segmentation results are displayed behind the person with a few frames' delay.

\section{Conclusions}

In this paper, we have developed an effective human region segmentation method for the live video segmentation system. The experimental results showed that our method is more effective than conventional methods for segmentation from 
dynamic backgrounds. There are three key contributions. The first contribution is the employing of the thermal feature. Second, we propose a new speed-up GraphCut algorithm by combining with the Bayesian estimation. The third contribution is a novel online learning method using accumulative histograms. The segmentation accuracy and speed are quite capable of the live video segmentation purpose. Our future work will be focused on further optimization of the algorithm and application of matting methods.

Acknowledgement: We express our thanks to Prof. H. Harashima of the University of Tokyo for his discussions.

\section{References}

1. Criminisi, A., Cross, G., Blake, A., Kolmogorov, V.: Bilayer segmentation of live video. In: Computer Vision and Pattern Recognition, vol. 1, pp. 53-60 (2006)

2. Sun, J., Zhang, W., Tang, X., Shum, H.-Y.: Background cut. In: Leonardis, A., Bischof, H., Pinz, A. (eds.) ECCV 2006. LNCS, vol. 3952, pp. 628-641. Springer, Heidelberg (2006)

3. Yasuda, K., Naemura, T., Harashima, H.: Thermo-key: human region segmentation from video. Computer Graphics and Applications, 26-30 (January-February 2004)

4. Boykov, Y., Jolly, M.: Interactive graph cuts for optimal boundary and region segmentation of objects in N-D images. In: IEEE International Conference on Computer Vision, vol. 1, pp. 105-112 (2001)

5. Rother, C., Kolmogorov, V., Blake, A.: Grabcut: interactive foreground extraction using iterated graph cuts. In: SIGGRAPH, vol. 23, pp. 309-314 (2004)

6. Incorp., A.S.: Adobe photoshop user guide (2002)

7. Mortensen, E., Barrett, W.: Intelligent scissors for image composition. In: Computer graphics and interactive techniques, pp. 191-198 (1995)

8. Kolmogorov, V., Criminisi, A., Blake, A., Cross, G., Rother, C.: Bi-layer segmentation of binocular stereo video. In: Computer Vision and Pattern Recognition, pp. 407-414 (2005)

9. Boykov, Y., Kolmogorov, V.: An experimental comparison of min-cut/max-flow algorithms for energy minimization in vision. IEEE Transactions on Pattern Analysis and Machine Intelligence, 1124-1137 (2004)

10. Implementation of Bilayer Segmentation of Live Video, http://vision.caltech.edu/projects/yiw/FgBgSegmentation/ 